\title{
Heart failure with recovered ejection fraction: Clinical characteristics, determinants and prognosis. CARDIOCHUS-CHOP registry
}

\author{
Rosa Agra Bermejo ${ }^{1}$, Eva González Babarro ${ }^{2}$, J. Nicolás López Canoa ${ }^{1}$, \\ Alfonso Varela Román ${ }^{1}$, Inés Gómez Otero ${ }^{1}$, Marcos Oro Ayude ${ }^{1}$, Pablo Parada Vázquez ${ }^{1}$, \\ Isabel Gómez Rodríguez ${ }^{2}$, Oscar Díaz Castro ${ }^{2}$, José Ramón Gónzalez-Juanatey ${ }^{1}$ \\ ${ }^{1}$ Servicio de Cardiología y Unidad Coronaria, Hospital Clínico Universitario \\ de Santiago de Compostela, A Coruña, Spain \\ ${ }^{2}$ Servicio de Cardiología y Unidad Coronaria, Hospital Montecelo, Pontevedra, Pontevedra, Spain
}

\begin{abstract}
Background: The magnitude and the prognostic impact of recovering left ventricular ejection fraction $(L V E F)$ in patients with heart failure (HF) and systolic dysfunction is unclear. The aim of this study was to evaluate the clinical characteristics and prognosis of patients with HFrecEF in an HF population. Methods: 449 consecutive patients were selected with the diagnosis of HF and an evaluation of LVEF in the 6 months prior to selection who were referred to two HF units. Patients with systolic dysfunction were only considered if a second echocardiogram was performed during the follow-up.

Results: At the time of diagnosis, 207 patients had LVEF > 40\% (HFDEF) and 242 had LVEF $\leq 40 \%$ (HFrEF). After 1 year, the LVEF was re-evaluated in all 242 patients with a LVEF $\leq 40 \%$ : in 126 (52\%), the second LVEF was $>40 \%$ (HFrecEF), and the remaining $116(48 \%$ ) had LVEF $\leq 40 \%$ (HFrEF). After $1800 \pm 900$ days of follow-up patients with recovered LVEF had a significantly lower mortality rate (HFpEF vs. HFrecEF: hazard ratio [HR] = 2.286, 95\% confidence interval [95\% CI] 1.264-4.145, $p=0.019$; HFrEF vs. HFrecEF: HR = 2.222, 95\% CI 1.189-4.186, $p<0.001$ ) and hospitalization rate (HFpEF vs. HFrecEF: $H R=1.411,95 \%$ CI 1.046-1.903, $p=0.024$; HFrEF vs. HFrecEF: $H R=1.388,95 \%$ CI 1.002-1.924, $p=0.049$ ). The following are predictors of LVEF recovery: younger age, lower functional class, treatment with renin-angiotensin-aldosterone system inhibitors and beta-blockers, absence of defibrillator use, and non-ischemic etiology.

Conclusions: Patients with HF and reduced LVEF who were re-evaluated after 1 year, had significant improvement in their LVEF and had a more favourable prognosis than HF with preserved and reduced ejection fraction. (Cardiol J 2018; 25, 3: 353-362)
\end{abstract}

Key words: heart failure, recovered, left ventricular ejection fraction

\section{Introduction}

The main terminology used to describe heart failure (HF) is historical and is based on left ventricular ejection fraction (LVEF) measurement which determines different etiologies, demographics, comorbidities, and response to therapies [1]. The 2012 European Society of Cardiology (ESC)
Heart Failure Guidelines classified patients with $\mathrm{HF}$ using a cut-off point $<50 \%$ for $\mathrm{HF}$ with reduced LVEF (HFrEF) and $\geq 50 \%$ for HF with preserved LVEF (HFpEF), but the therapeutic recommendations were specific only for patients with LVEF $\leq 40 \%[2,3]$.

The new 2016 ESC Heart Failure Guidelines propose a new classification according to the level

Address for correspondence: Rosa Agra Bermejo, MD, PhD, Servicio de Cardiología y Unidad Coronaria, Hospital Clínico Universitario de Santiago de Compostela, Travesía da Choupana s/n, 15700- Santiago de Compostela (A Coruña), tel: +34650519163 or +34981950242 , e-mail: rosinagra@msn.com 
of LVEF, as follows: $\mathrm{HFrEF}$ is $<40 \%$, mid-range $\mathrm{HF}$ is an LVEF range $40-49 \%$, and $\mathrm{HFpEF}$ is $\geq 50 \%$ [4]. Currently, there is limited information available about the clinical, prognostic, and therapeutic implications of this classification [5].

In addition to clinical characterization and therapeutic properties, HF classification based on LVEF allows the identification of groups of patients with differences in prognosis. HFrEF patients, in particular, have a higher mortality rate, but a similar mortality rate between $\mathrm{HFrEF}$ and $\mathrm{HFpEF}$ has been described $[3,6]$.

Left ventricular ejection fraction is known to change during the natural course of the disease and $\mathrm{HF}$ with a recovered ejection fraction (HFrecEF) has recently been identified. HFrecEF includes patients who initially present with depressed LVEF and who recover to a value $>40 \%$. Some studies suggest that this new type of HF may be a different clinical entity with a particular pattern of biomarkers, comorbidities, and a better mid-to-long-term prognosis, suggesting that these patients may have entered into a phase of disease remission [7-14].

The aim of this study was to evaluate the clinical characteristics and prognosis of HFrecEF patients within an HF population.

\section{Methods}

\section{Study population}

This was a retrospective cohort study of 596 consecutive patients who were referred for first time to a HF unit in the Cardiology Department at two Spanish hospitals between September 2007 and January 2014. The study was approved by the local bioethical committee and all patients gave their informed consent. HF diagnostic was made in accordance with the 2012 ESC Heart Failure Guidelines [2].

Patients excluded were those with specific infiltrative or restrictive cardiomyopathies, HF due to adult congenital heart disease and patients with primary right-sided disease. Thirty-seven patients were excluded because they did not have LVEF data in the 6 months prior to inclusion and 110 patients with HFrEF were excluded because they were not assessed for LVEF at follow-up. Thus, 449 patients in stable conditions were included in the analyses and classified according to their LVEF, as follows: 207 with HFpEF (LVEF > 40\%) and 242 with HFrEF (LVEF $\leq 40 \%)$.

After a mean of 1 year (median, 1.51 years) from the beginning of the study, all included HFrEF

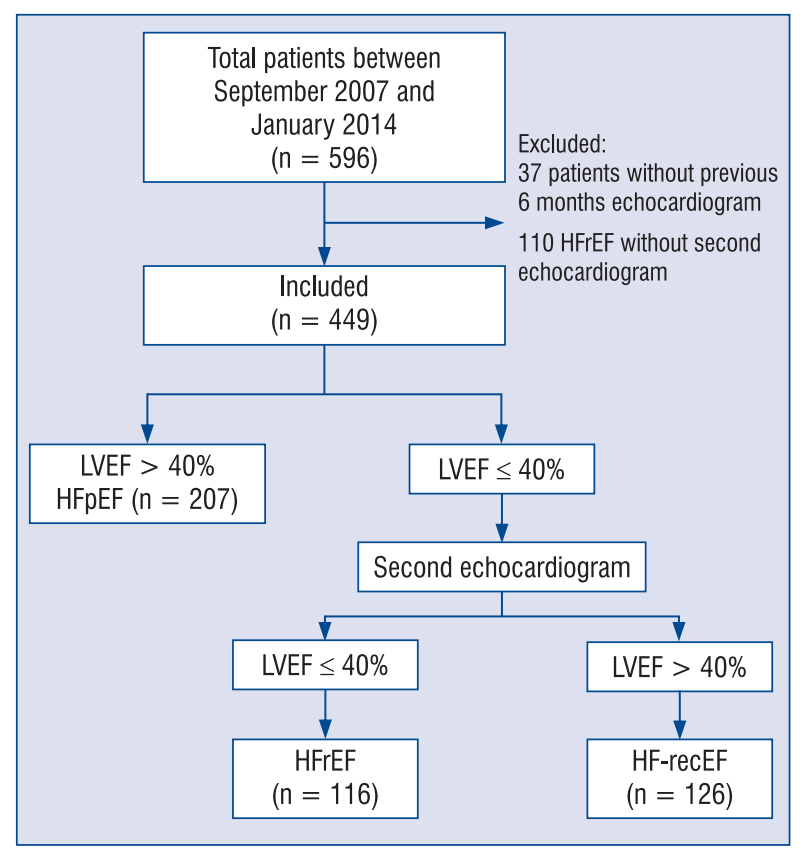

Figure 1. Flowchart of patients included in the study; HFrEF - heart failure with remained reduced left ventricular ejection fraction; HFpEF - heart failure with preserved left ventricular ejection fraction; HFrecEF heart failure with recovered left ventricular ejection fraction; LVEF — left ventricular ejection fraction.

patients had a second echocardiographic assessment performed in a situation of clinical stability and optimal medical treatment. After this control, 126 patients had LVEF > 40\% (HFrecEF) and 116 maintained a LVEF $\leq 40 \%$ (HFrEF) (Fig. 1).

For all included patients, detailed information was gathered from their medical history and appropriate physical examination information was obtained from their charts, this information was recorded in a database. In addition, blood samples were obtained for local laboratory analysis and LVEF was estimated using the Simpson method according to current international recommendations [15]. HF was defined as being ischemic in etiology if any of the following criteria were satisfied: prior admission because of an acute coronary event (acute myocardial infarction or unstable angina), prior surgical or percutaneous myocardial revascularization, presence of myocardial infarction on electrocardiogram or echocardiogram, or significant coronary disease detected by angiography. Interventions before inclusion in the study, such as device implantation (implantable cardioverter defibrillator [ICD] and/ /or cardiac resynchronization therapy [CRT]), and previous revascularization were recorded. 


\section{Follow-up}

All patients were followed for a median of $1800 \pm 900$ (mean \pm standard deviation) days. The primary endpoints were death or hospitalization for any cause. Interventions with a prognostic impact on the course of the disease (device implantation, revascularization or cardiac transplantation) were recorded.

\section{Statistical analysis}

Patient characteristics were analyzed using statistical inference as follows: $\chi^{2}$ test, Student's t-test, ANOVA, Fisher exact test, and the Kruskal-Wallis test.

Event-free survival curves were calculated using the Kaplan-Meier method, and differences between the curves were evaluated using the log-rank test. Univariate and multivariate Cox regression analyses were used to calculate the estimated hazard ratio (HR) with a $95 \%$ confidence interval (CI) in where appropriate. The variables were entered into a multivariate model for factors with a $\mathrm{p}$ value of $\leq 0.05$ in the univariate analysis. A multivariate analysis, such as a binary logistic regression, was used to determine the variables with independent prognostic significance in the LVEF recovery. This analysis included variables that showed significant differences in the univariate analysis. The Statistical Package for Social Science (SPSS) for Windows, version 15.0 (software SPSS Inc.; Chicago, Illinois, United States) was used for all statistical analysis. A p value of $<0.05$ was considered to be statistically significant

\section{Results}

\section{Baseline characteristics}

When the baseline characteristics of $\mathrm{HpEF}$, HFrEF and HFrecEF patients were analyzed, observations revealed statistically significant differences in age, sex, smoking, previous myocardial infarction, previous percutaneous revascularization, previous device (ICD/CRT) implantation, systolic blood pressure, New York Heart Association (NYHA) class, heart rate, QRS duration, presence of left bundle branch block, pro B-type natriuretic peptide (proBNP) levels, HF etiology, and treatment with angiotensin converting enzyme inhibitors (ACEIs), beta-blockers, aldosterone antagonists, statins, acetylsalicylic acid (ASA), clopidogreland oral anticoagulation (Table 1).

Patients with HFrecEF were younger, had a lower percentage of previous revascularization and use of devices, with a less advanced functional class (no patient had NYHA IV), and a lower prevalence of ischemic etiology compared with $\mathrm{HFpEF}$ and HFrEF. These patients had greater use of ACEIs and less use of anticoagulants and anti-platelet medications.

Characteristics of patients not re-evaluated were also analyzed (Suppl. Table 1) and it was found that these patients were more likely to be older, with higher levels of proBNP and lower levels of hemoglobin and were treated more frequently with diuretics and less with optimal medical treatment compared with revaluated patients.

\section{LVEF values}

Table 1 shows the mean baseline LVEF in the three groups. The mean recovery was $+10.31 \pm$ $\pm 12.95 \%$ SD (range, $-18 \%$ to $+50 \%$ ). The LVEF did not change or worsened in $61(25.4 \%)$ patients, and recovery was between $0 \%$ and $10 \%$ in 64 (26.6\%) patients, between $10 \%$ and $20 \%$ in 60 $(24.6 \%)$ patients, and $>20 \%$ in $57(23.4 \%)$ patients.

\section{Follow-up}

The 449 patients were followed up for a median of $1800 \pm 900$ days. During this time, statistically significant differences were observed for device implantation, which was greater in patients who maintained a reduced LVEF. For these patients, there were 27 defibrillator implants in HFrEF patients (20.1\%) compared with 3 in HFrecEF patients (2.8\%; $\mathrm{p}<0.001), 19$ resynchronizer implants in HFrEF patients (14.2\%) compared with 3 in HFrecEF patients $(2.8 \% ; \mathrm{p}<0.001)$, and 13 defibrillator-resynchronizer implants in HFrEF patients $(9.7 \%)$ compared with 1 in HFrecEF patients $(0.9 \%$; $\mathrm{p}<0.001)$.

There were $165(36.7 \%)$ patients in this study who died. There were $100(50.3 \%)$ deaths in the $\mathrm{HFpEF}$ group, $43(42.2 \%)$ in the HFrEF group, and $22(20.2 \%)$ in the HFrecEF group $(\mathrm{p}<0.01)$. Survival time was higher in HFrecEF patients $(5.2 \pm 1.94$ years $)$ than in HFrEF patients $(5.65 \pm$ \pm 2.25 years $)$ and $\mathrm{HFpEF}$ patients $(4.97 \pm 2.46$ years; $\mathrm{p}<0.001$ ).

During follow-up, $312(69.4 \%)$ patients required hospitalization; 160 (80.4\%) in the $\mathrm{HFpEF}$ group, $84(82.4 \%)$ in the HFrEF group, and 68 $(62.4 \%)$ in the HFrecEF group ( $<<0.001)$. Time free from hospitalization was higher in HFrecEF patients ( $3.46 \pm 2.26$ years), compared to $\mathrm{HFpEF}$ patients $(2.71 \pm 2.49$ years $)$ and $\mathrm{HFrEF}$ patients $(2.56 \pm 2.24$ years; $\mathrm{p}<0.001)$.

Kaplan-Meier curves and log-rank tests for death and hospitalization showed statistically significant differences between the three groups ( $p<0.005$ for both comparisons; Fig. 2A, B). 
Table 1. Baseline characteristics of the population.

\begin{tabular}{|c|c|c|c|c|}
\hline & HFpEF (n = 207) & HFrEF $(n=116)$ & HFrecEF $(n=126)$ & $\mathbf{P}$ \\
\hline \multicolumn{5}{|l|}{ Demographic and medical history } \\
\hline Age [years] & $71 \pm 10$ & $66 \pm 12$ & $63 \pm 12$ & $<0.001$ \\
\hline Male & $120(58 \%)$ & $89(76.7 \%)$ & $92(73 \%)$ & $<0.001$ \\
\hline \multicolumn{5}{|l|}{ Medical history: } \\
\hline Tobacco use: & & & & $<0.001$ \\
\hline Current & $138(66.7 \%)$ & $56(48.3 \%)$ & $61(48.4 \%)$ & \\
\hline Former & $8(3.9 \%)$ & $22(19 \%)$ & $24(19 \%)$ & \\
\hline Never & $61(29.1 \%)$ & $38(32.8 \%)$ & $41(32.5 \%)$ & \\
\hline Alcohol & $35(17 \%)$ & $25(21.6 \%)$ & $40(35.7 \%)$ & 0.007 \\
\hline Hypertension & $133(64.3 \%)$ & $33(54.3 \%)$ & $69(54.8 \%)$ & 0.11 \\
\hline Diabetes mellitus & $67(32.4 \%)$ & $39(33.6 \%)$ & $41(32.6 \%)$ & 0.97 \\
\hline Peripheral arterial disease & $13(6.3 \%)$ & $9(7.8 \%)$ & $7(5.3 \%)$ & 0.51 \\
\hline Previous stroke & $11(5.3 \%)$ & $7(6 \%)$ & $8(6.3 \%)$ & 0.91 \\
\hline Previous ACS & $31(15 \%)$ & $36(31 \%)$ & $13(10.3 \%)$ & $<0.001$ \\
\hline Previous HF admission & $146(70.5 \%)$ & $79(68.1 \%)$ & $81(64.3 \%)$ & 0.495 \\
\hline Previous stent placement & $27(13 \%)$ & $29(25 \%)$ & $12(9.5 \%)$ & 0.012 \\
\hline Previous CABG & $21(10.1 \%)$ & $14(12.1 \%)$ & $9(7.1 \%)$ & 0.78 \\
\hline Previous defibrillator & $2(1 \%)$ & $18(15.5 \%)$ & $3(2.8 \%)$ & $<0.001$ \\
\hline Previous CRT & $1(0.5 \%)$ & $4(3.4 \%)$ & $2(1.6 \%)$ & 0.003 \\
\hline \multicolumn{5}{|c|}{ Physical examination and supplementary tests } \\
\hline NYHA class: & & & & 0.015 \\
\hline I & $38(19.8 \%)$ & $16(13.8 \%)$ & $25(19.8 \%)$ & \\
\hline II & $120(58 \%)$ & $72(62.1 \%)$ & $90(71.4 \%)$ & \\
\hline III & $48(23.2 \%)$ & $25(21.6 \%)$ & $11(8.7 \%)$ & \\
\hline IV & $1(0.5 \%)$ & $3(2.6 \%)$ & $0(0 \%)$ & \\
\hline Baseline HR [bpm] & $75.89 \pm 19.37$ & $74.69 \pm 14.77$ & $73.31 \pm 16.65$ & 0.43 \\
\hline Baseline SBP [mmHg] & $132.9 \pm 21.81$ & $120.69 \pm 19.8$ & $128.64 \pm 22.12$ & $<0.001$ \\
\hline Baseline DBP [mmHg] & $78.63 \pm 14.38$ & $76.64 \pm 13.32$ & $78.99 \pm 14.491$ & 0.37 \\
\hline Body mass index $\left[\mathrm{kg} / \mathrm{m}^{2}\right]$ & $32 \pm 21$ & $27 \pm 5$ & $28 \pm 4$ & 0.017 \\
\hline ProBNP $[\mathrm{pg} / \mathrm{m}]$ & $1906.21 \pm 2123.23$ & $2337.25 \pm 24490.04$ & $1210.40 \pm 1293.23$ & 0.001 \\
\hline Creatinine $[\mathrm{mg} / \mathrm{dL}]$ & $1.17 \pm 0.38$ & $1.17 \pm 0.39$ & $1.13 \pm 0.37$ & 0.61 \\
\hline Hemoglobin $[\mathrm{g} / \mathrm{dL}]$ & $13.7 \pm 1.09$ & $13.3 \pm 1.61$ & $13.5 \pm 1.71$ & 0.90 \\
\hline Sodium [meq/L] & $138.4 \pm 3.4$ & $138.5 \pm 3.0$ & $138.3 \pm 3.0$ & 0.90 \\
\hline Anemia & $58(28 \%)$ & $24(21 \%)$ & $27(21 \%)$ & 0.21 \\
\hline Heart rhythm: & & & & $<0.001$ \\
\hline $\mathrm{SR}$ & $81(39.5 \%)$ & $75(65.2 \%)$ & $85(68 \%)$ & \\
\hline $\mathrm{AF}$ & $104(50.7 \%)$ & $27(23.5 \%)$ & $28(22.4 \%)$ & \\
\hline LBBB & $23(11.6 \%)$ & $38(37.3 \%)$ & $20(18.3 \%)$ & $<0.001$ \\
\hline QRS duration [ms] & $114.4 \pm 34.2$ & $138.4 \pm 36.6$ & $120.2 \pm 30.2$ & $<0.001$ \\
\hline Baseline LVEF [\%] & $56.4 \pm 9.5$ & $29.1 \pm 7.3$ & $31.3 \pm 6.1$ & $<0.001$ \\
\hline \multicolumn{5}{|l|}{ Etiology and baseline treatment } \\
\hline \multicolumn{5}{|l|}{ Etiology: } \\
\hline Ischemic heart disease & $44(21.3 \%)$ & $57(49.1 \%)$ & $25(19.8 \%)$ & \\
\hline Valvular & $41(19.8 \%)$ & $10(8.6 \%)$ & $10(7.9 \%)$ & \\
\hline Hypertrophic cardiomyopathy & $6(2.9 \%)$ & $9(7.8 \%)$ & $7(5.6 \%)$ & \\
\hline Enolic & $17(8.2 \%)$ & $7(6.2 \%)$ & $26(20 \%)$ & \\
\hline Hypertension & $58(28 \%)$ & $19(16.4 \%)$ & $34(27 \%)$ & \\
\hline Others & $43(20 \%)$ & $14(12 \%)$ & $78(19 \%)$ & $<0.001$ \\
\hline Diuretics & $170(82.1 \%)$ & $96(82.2 \%)$ & $92(73 \%)$ & 0.27 \\
\hline ACEls or ARBs & $158(76.3 \%)$ & $102(87.9 \%)$ & $113(90.4 \%)$ & $<0.001$ \\
\hline Beta-blockers & $119(57.1 \%)$ & $97(83.6 \%)$ & $98(77.8 \%)$ & $<0.001$ \\
\hline MRA & $81(39.1 \%)$ & $63(54.3 \%)$ & $52(41.3 \%)$ & 0.025 \\
\hline Statins & $74(35.7 \%)$ & $59(51.8 \%)$ & $48(38.4 \%)$ & 0.017 \\
\hline Oral anticoagulation & $103(49.8 \%)$ & $40(34.5 \%)$ & $39(31 \%)$ & 0.001 \\
\hline ASA & $69(33.3 \%)$ & $48(41.4 \%)$ & $50(39.7 \%)$ & 0.021 \\
\hline Clopidogrel & $19(9.2 \%)$ & $23(19.8 \%)$ & $15(11.9 \%)$ & 0.007 \\
\hline
\end{tabular}

ACS - acute coronary syndrome; ACEls - angiotensin-converting enzyme inhibitors; AF — atrial fibrillation; ASA — acetylsalicylic acid; ARBs - angiotensin receptor blockers; CABG - coronary artery bypass graft; CRT — cardiac resynchronization therapy; DBP — diastolic blood pressure; HF - heart failure; HFrEF - heart failure with remained reduced left ventricular ejection fraction; HFpEF - heart failure with
preserved left ventricular ejection fraction; HFrecEF — heart failure with recovered left ventricular ejection fraction; HR - heart rate; LBBB preserved left ventricular ejection fraction; HFrecEF - heart failure with recovered left ventricular ejection fraction; HR - heart rate; LBBB -
left bundle branch block; LVEF — left ventricular ejection fraction; MRA - mineralcorticoid receptor antagonists; NYHA - New York Heart Association; proBNP — pro-B-type natriuretic peptide; SBP — systolic blood pressure; SR — sinus rhythm 


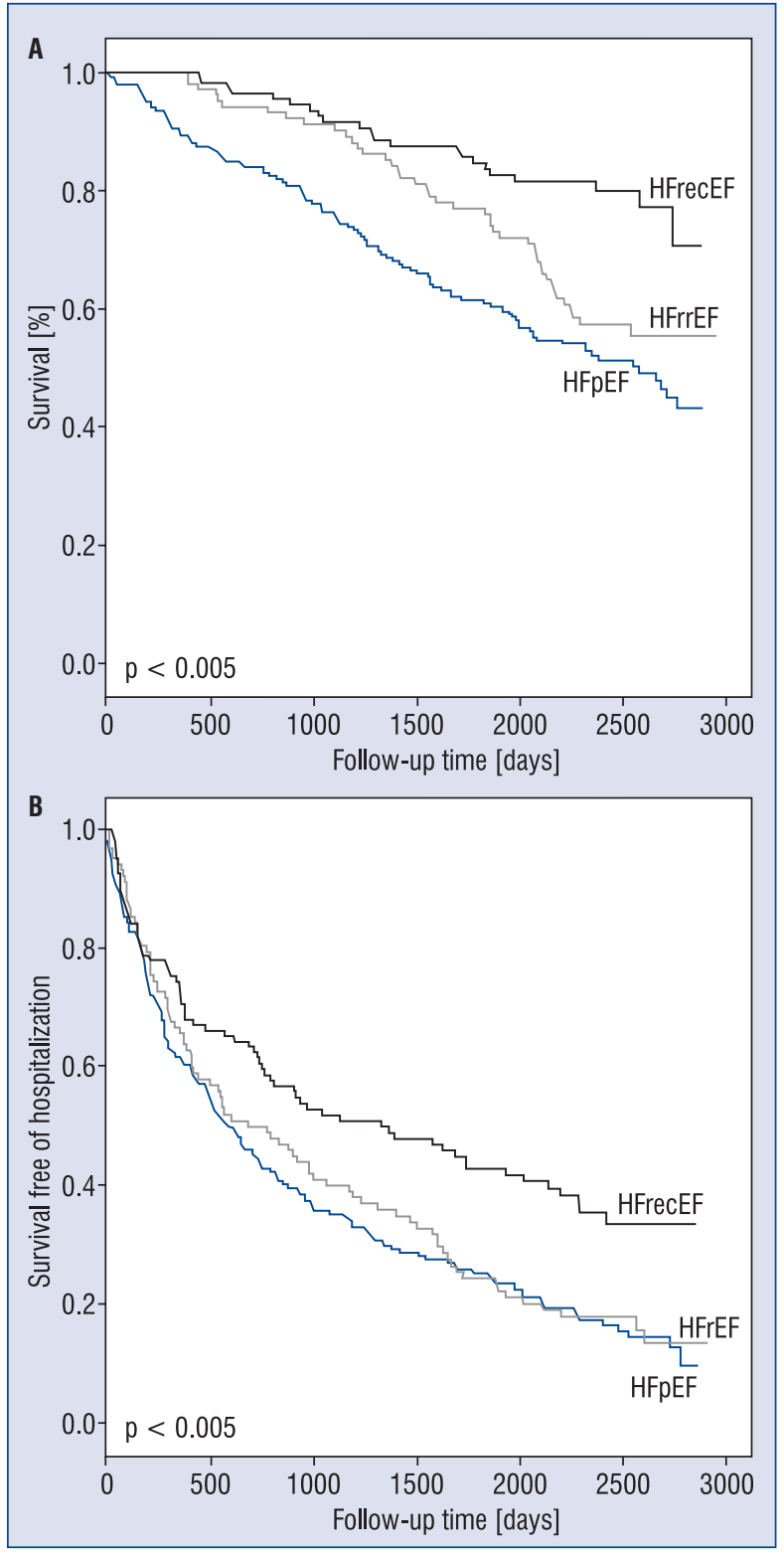

Figure 2. Survival curve in terms of mortality $(\mathbf{A})$ and hospitalization (B) in heart failure with remained reduced left ventricular ejection fraction (HFrEF), heart failure with preserved left ventricular ejection fraction (HFpEF) and heart failure with recovered left ventricular ejection fraction (HFrecEF).

The patient prognosis was also evaluated according to the percentage of change in LVEF. The subgroup that recovered LVEF > 20\% had a higher survival rate, although this increase was not statistically significant $(\mathrm{p}=0.08)$, and had a lower percentage of hospitalizations $(\mathrm{p}<0.001$; Fig. 3A, B).

The group of not re-evaluated patients had higher mortality $(43,1 \%)$ compared with the re-

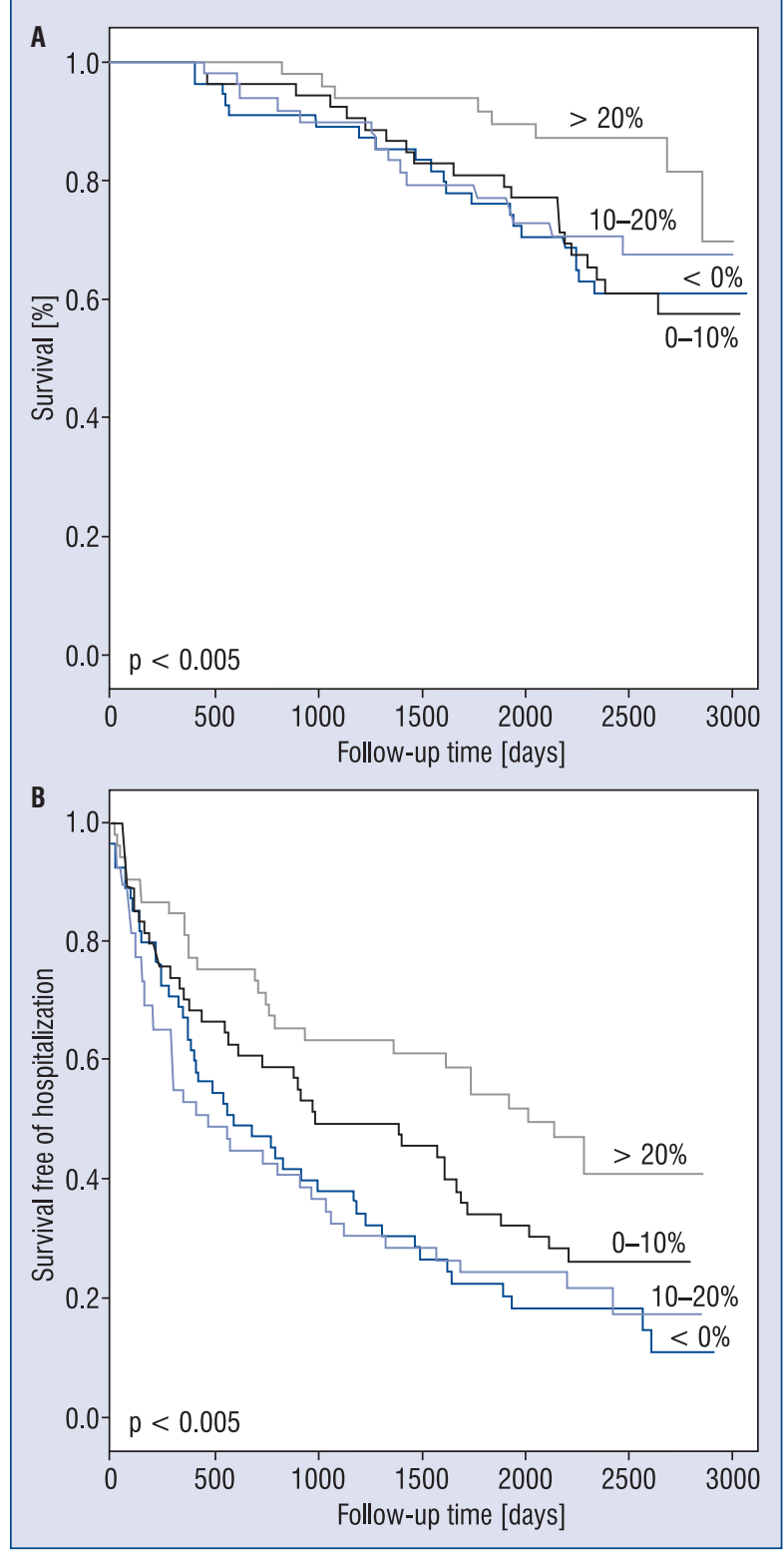

Figure 3. Survival curve in terms of mortality (A) and hospitalization (B) as a function of the percentage of recovered left ventricular ejection fraction.

evaluated group $(8.5 \%, \mathrm{p}<0.001)$ and had no differences in terms of readmissions (63.3\% vs. $72 \%, \mathrm{p}=0.14)($ Fig. 4).

\section{Independent predictors of mortality}

An analysis was performed using Cox regression to identify independent predictors of mortality. Only the variables with statistical significance were included in the univariate analysis. The variables used were HFrecEF, age, sex, smoking, previous myocardial infarction or revascularization, previ- 


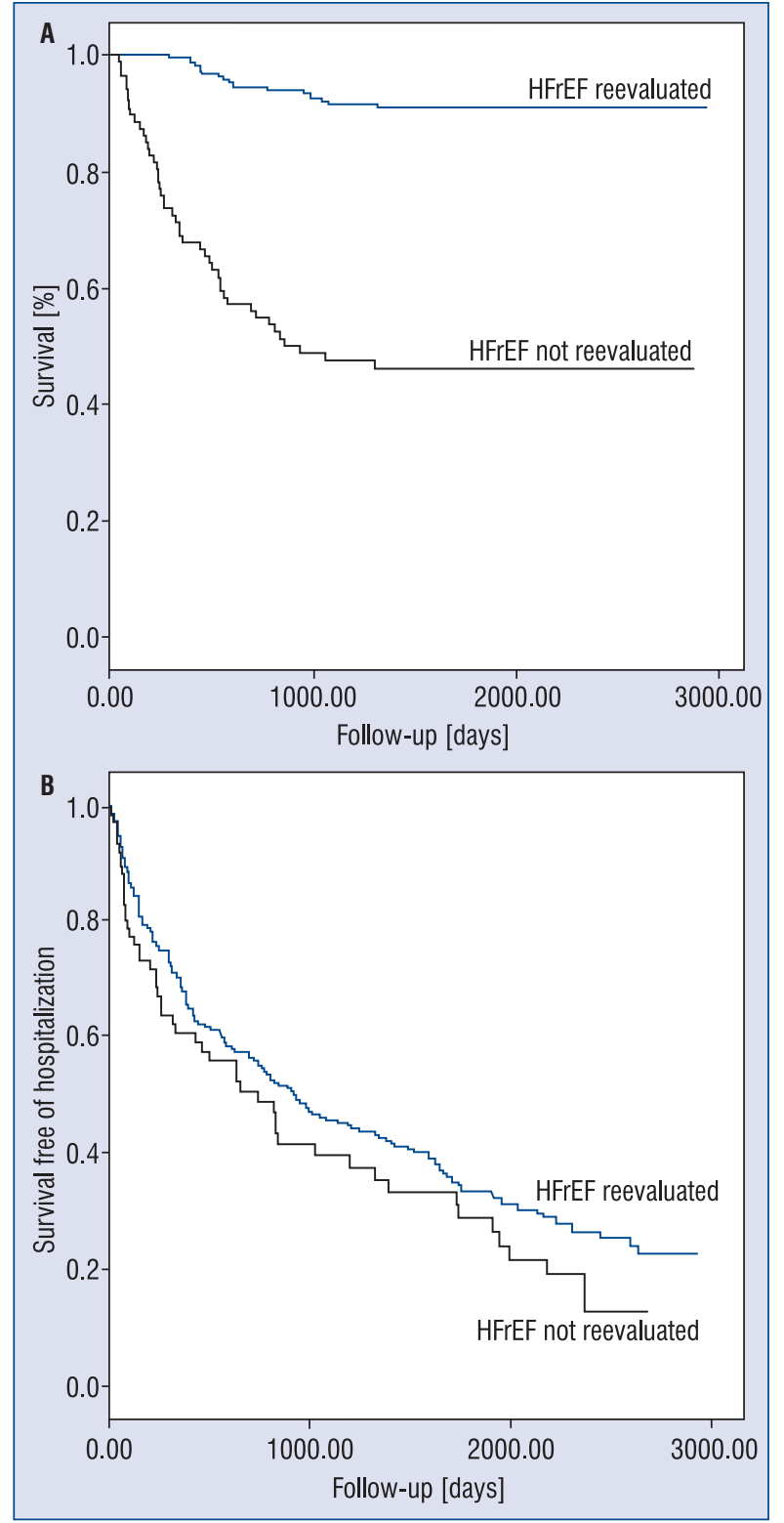

Figure 4. Survival curves in terms of mortality (A) and hospitalization (B) in heart failure with remained reduced left ventricular ejection fraction (HFrEF) revaluated and not revaluated patients.

ous device implantation, systolic blood pressure, NYHA III-IV, heart rhythm, QRS duration, left bundle branch block, proBNP levels, HF etiology, ACEIs, beta-blockers, mineralocorticoid receptor antagonists, statins, ASA, clopidogrel, and oral anticoagulation.

Left ventricular ejection fraction recovery continued to be an independent prognostic variable for mortality. Thus, HFpEF patients had a risk of death that was 2.28-times higher than that of patients with HFrecEF, and HFrEF 2.2-times higher than that of patients with HFrecEF.
Table 2. Indepedent predictors of mortality.

\begin{tabular}{lccc}
\hline & $\begin{array}{c}\text { Hazard } \\
\text { ratio }\end{array}$ & $\begin{array}{c}95 \% \\
\text { confidence } \\
\text { interval }\end{array}$ & P \\
\hline Age & 1.051 & $1.028-1.075$ & $<0.001$ \\
proBNP & 1.000 & $1.000-1.000$ & $<0.001$ \\
HFpEF & 2.286 & $1.264-4.145$ & 0.019 \\
$\begin{array}{l}\text { vs. HFrecEF } \\
\text { HFrEF }\end{array}$ & 2.222 & $1.189-4.186$ & $<0.001$ \\
vs. HFrecEF & & & \\
\hline
\end{tabular}

HFrEF - heart failure with remained reduced left ventricular ejection fraction; HFpEF - heart failure with preserved left ventricular ejection fraction; HFrecEF - heart failure with recovered left ventricular ejection fraction; proBNP — pro-B-type natriuretic peptide

Table 3. Independent predictors of hospitalization.

\begin{tabular}{lccc}
\hline & $\begin{array}{c}\text { Hazard } \\
\text { ratio }\end{array}$ & $\begin{array}{c}95 \% \\
\text { confidence } \\
\text { interval }\end{array}$ & P \\
\hline Age & 1.014 & $1.003-1.024$ & 0.011 \\
NYHA III-IV & 1.541 & $1.166-2.037$ & 0.002 \\
$\begin{array}{l}\text { HFpEF } \\
\text { vs. HFrecEF }\end{array}$ & 1.411 & $1.046-1.903$ & 0.024 \\
$\begin{array}{l}\text { HFrEF } \\
\text { vs. HFrecEF }\end{array}$ & 1.388 & $1.002-1.924$ & 0.049 \\
\hline
\end{tabular}

HFrEF - heart failure with remained reduced left ventricular ejection fraction; HFpEF - heart failure with preserved left ventricular ejection fraction; HFrecEF - heart failure with recovered left ventricular ejection fraction; NYHA - New York Heart Association

Other independent variables with prognostic influence were age and proBNP level (Table 2).

\section{Independent predictors of hospitalization}

The same variables as in the analysis performed for mortality were used in this analysis for hospitalization. The LVEF recovery remained an independent prognostic variable on the hospitalization-free survival, compared with $\mathrm{HFpEF}$ and HFrEF.

Other independent prognostic variables for hospitalization-free survival were age and NYHA categories III and IV (Table 3).

\section{Independent prognostic factors for LVEF recovery}

A multivariate analysis was performed to determine which variables independently predict LVEF recovery. The variables that were significantly different were initially compared between the HFrEF and HFrecEF groups, as follows: age, sex, alcohol consumption, ischemic etiology, prior 
Table 4. Independent prognostic factors of left ventricular ejection fraction recovery.

\begin{tabular}{lccc}
\hline & $\begin{array}{c}\text { Hazard } \\
\text { ratio }\end{array}$ & $\begin{array}{c}95 \% \\
\text { confidence } \\
\text { interval }\end{array}$ & P \\
\hline Age & 0.957 & $0.939-0.976$ & $<0.001$ \\
NYHA III-IV & 0.422 & $0.199-0.893$ & 0.024 \\
ACEls and BB & 2.126 & $1.349-3.349$ & 0.001 \\
Ischemic & 0.385 & $0.224-0.662$ & 0.005 \\
etiology & & & \\
ICD & 0.285 & $0.02-0.990$ & 0.048 \\
Constant & 2.428 & & 0.270 \\
\hline
\end{tabular}

ACEls - angiotensin-converting enzyme inhibitors; BB - beta-blockers; ICD — implantable cardioverter-defibrillator; NYHA — New York Heart Association

defibrillator, NYHA III-IV, systolic blood pressure, beta blockers, aldosterone antagonists, statins, and defibrillator and/or resynchronizer implantation during follow-up.

The NYHA class III-IV, treatment with ACEIs and beta-blockers, ischemic etiology and previous defibrillator implantation were found to be predictors of recovery and non-recovery (Table 4).

\section{Discussion}

The data showed that the HFrecEF population has a different clinical profile (demographics, comorbidities, and symptom severity) compared with $\mathrm{HFrEF}$ and $\mathrm{HFpEF}$ populations. Patients who experienced LVEF recovery have an excellent prognosis in terms of mortality and hospitalization, particularly in those patients with a LVEF recovery higher than $20 \%$.

A younger age, better NYHA functional class, treatment with ACEIs and beta-blockers, nonischemic etiology, and the previous ICD implantation follow-up were independent predictors of LVEF recovery.

The present results suggest that HFrecEF represents a separate clinical entity with clinical and prognosis differences compared with the classic groups, and it is also believed that it is a distinct category along the HF continuum. These observations indicate that LVEF may be considered to be a guide-element for therapy optimization and follow-up in the same way that was proposed for natriuretic peptides [4].

Punnoose et al. [16] reported that nearly $30 \%$ of patients in their registry, who had normal LVEF on echocardiography, previously had a reduced LVEF, and they may represent a sub-group of $\mathrm{HF}$ patients with favourable cardiac and vascular remodeling [11].

According to the "cancer model", HFrecEF patients could have a process of disease remission because of reverse remodeling that is characterized by an improvement in LVEF and a more favorable clinical profile, but without complete cardiac recovery. However, these patients continued to experience significant HF symptoms, signs, and clinical complications, including several cardiac hospitalizations and mortality during the follow-up [12]. Moreover, clinical deterioration and recurrence of cardiac dysfunction when medical and device support were discontinued was described $[17,18]$. Basuray et al. [7] showed that in patients with HFrecEF, that there is persistent neurohormonal activation, increased oxidative stress, and cardiomyocyte injury and stress despite apparent LVEF recovery. Event-free survival in the HFrecEF group was better than that in the $\mathrm{HFrEF}$ and HFpEF groups but the symptoms and the risk of hospitalization were similar to those of the HFpEF population. In this study, statistically significant differences were not observed in the prognosis of patients with $\mathrm{HFrEF}$ or HFpEF, which is in agreement with previous publications from the present group [6, 19].

In the baseline HFrEF population a mean LVEF increase of $10 \%$ was observed. This finding was consistent with results described by Wilcox et al. [20]. From an individual patient perspective, a question arises from present results as to whether it is better to use LVEF higher than $40 \%$ or the LVEF recovery magnitude as in the index. A better prognosis was seen in patients with an increase in LVEF that was greater than $20 \%$, which is similar to that reported in the super-responder patients treated with a cardiac-resynchronization device [21]. Thus, the LVEF baseline level and the recovery magnitude can play complementary roles. The indication for battery replacement in patients with a cardiac resynchronization device who had also recovered LVEF is questioned in recent ESC Heart Failure Guidelines [4]. This is an important issue from a clinical and economic perspective. Despite the absence of relevant publications regarding this recommendation, the present results describe a patient population that can be affected and better prognoses can be seen in this $\mathrm{HFrecEF}$ population, reinforcing this guideline recommendation. Current knowledge is limited, so this decision needs to be individualized according to the clinical-demographic and socioeconomic determinants. 
If a CRT is indicated, it is necessary for patients to be receiving optimal medical therapy for at least 3 months and to maintain a reduced LVEF [4]. Results herein suggest that CRT implantation based on a remote LVEF assessment can overestimate the proportion of patients who require a CRT and islikely because of the LVEF recovery effect during follow-up after treatment optimization.

In this study, LVEF recovery was an independent predictor of mortality in a multivariate analysis. The mortality risk was 2.2-times higher in $\mathrm{HFpEF}$ patients and in patients who continued to have a reduced LVEF, compared with patients with HFrecEF (Table 2). Age and BNP levels were also independent predictors of mortality in multivariate analysis [22-25]. Additionally, LVEF recovery was an independent predictor of survival free of hospitalization compared with $\mathrm{HFpEF}$ and $\mathrm{HFrEF}$ patients (Table 3). This finding was not observed in Basuray et al. [7]; the differences between both patient populations may explain the differences in these results.

Five variables were identified as predictors of LVEF recovery and LVEF that remained depressed $(<40 \%)$. The probability of LVEF recovery was reduced with advancing age, as was previously described [23, 24]; the highest capacity for reversing remodeling in younger patients can influence this finding. It also also showed that patients with advanced NYHA functional classes had a lower probability of LVEF recovery [26].

In this study, treatment with ACEIs and betablockers was the strongest predictor of LVEF recovery. These drugs have shown a favorable effect on patient survival and decreased morbidity [27, 28 ] in patients with HFrEF, they also improve cardiac remodeling factors [29], which may contribute to explaining the LVEF recovery process.

The interaction between the HF ischemic etiology and the lower probability of LVEF recovery may be influenced by the presence of myocardial viability [30] and a limited number of ventricular viable segments compared with patients with $\mathrm{HF}$ resulting from primary dilated cardiomyopathy [20]. Moreover, the amount of the LVEF increase associated with medical therapy (ACEIs and betablockers) was lower in patients with ischemic cardiomyopathy compared with HF patients with non-ischemic etiologies [31]. Ischemic etiology was more frequent in the present group of patients who maintained HFrEF during the follow-up, and non-ischemic causes (hypertensive, alcoholic, and primary dilated cardiomyopathy) were more frequent in $\mathrm{HFrecEF}$ patients possibly because of the higher capacity for myocardial reverse remodeling that is associated with non-ischemic HF patients.

Finally, the previous ICD implantation was associated with a lower probability of LVEF recovery. The role of CRT on LVEF recovery is well known [21] and there is no evidence on the recovery in patients with ICD [32]. However, the results may be influenced by the present study characteristics with patients who had more advanced myocardial damage undergoing ICD implantation. This may significantly influence the finding that ICD implantation is a marker of non-recovery.

\section{Limitations of the study}

Heart failure classification according to the LVEF is based on a consensus, and there are discrepancies between different clinical guidelines and expert position papers $[4,25]$.

Left ventricular ejection fraction assessed by echocardiography was used to classify patients and for follow-up, and the technique variability in this method may have influenced these results, even though all the echo studies were performed by cardiologists with experience in cardiac imaging. This study focused on LVEF changes, and other cardiac structure and function parameters which were not assessed.

Patients were included in the registry during their first consultation in the indicated HF outpatient clinics, but there was no precise information on the duration of the HF process. The present findings are limited to a cohort of patients who had a chronic HF condition and LVEF re-evaluation after a 1-year period; a more prolonged follow-up period could increase the number of patients in the HF-recovered group, or patients with LVEF recovery early after treatment optimization could undergo deterioration during a more prolonged observation period.

Because of the relatively small size of our HFrecEF group treated with CRT, there is insufficient data to analyze predictors of device response or to determine the specific prognosis of CRT responders.

The proportion of patients without LVEF assessment after a 1-year follow-up may have influenced the present findings, although high mortality during this follow-up period (Suppl. Table 1, Fig. 4) identifies a group of patients with a poor prognosis, which would partially explain why they did not have an echocardiographic control.

The prospective follow-up of these patients is one of the strengths of this study. A retrospective selection and classification of the patients can be a limitation in similar, previously published studies [7]. 
The design of this study probably reduced patient misclassification and may offer more precise information on the real prevalence of HFrecEF.

\section{Conclusions}

In a cohort of patients with HFrEF, the recovery process identifies a group of patients with an improved prognosis compared with the patients who maintained a decreased LVEF during followup, particularly in those with a LVEF increase higher than 20\%. Treatment with ACEIs and beta-blockers along with the NYHA functional class were the strongest predictors of LVEF recovery. This study underscores the need for further investigation of the pathophysiologic characteristics associated with the recovery process, to better tailor therapy and extend the period of disease remission.

\section{Acknowledgements}

The present study was supported by Complejo Hospitalario Universitario de Santiago de Compostela (Santiago de Compostela, Spain) and cofounded by ISCIII-Subdirección General de Evaluación y Fomento de la Investigación el Fondo Europeo de Desarrollo Regional (FEDER).

\section{References}

1. Butler J, Fonarow GC, Zile MR, et al. Developing therapies for heart failure with preserved ejection fraction: current state and future directions. JACC Heart Fail. 2014; 2(2): 97-112, doi: 10.1016/j.jchf.2013.10.006, indexed in Pubmed: 24720916.

2. McMurray JJV, Adamopoulos S, Anker SD, et al. ESC Guidelines for the diagnosis and treatment of acute and chronic heart failure 2012: The Task Force for the Diagnosis and Treatment of Acute and Chronic Heart Failure 2012 of the European Society of Cardiology. Developed in collaboration with the Heart Failure Association (HFA) of the ESC. Eur Heart J. 2012; 33(14): 1787-1847, doi: 10.1093/eurheartj/ehs104, indexed in Pubmed: 22611136.

3. Yusuf S, Pfeffer MA, Swedberg K, et al. Effects of candesartan in patients with chronic heart failure and preserved left-ventricular ejection fraction: the CHARM-Preserved Trial. Lancet. 2003; 362(9386): 777-781, doi: 10.1016/S0140-6736(03)14285-7, indexed in Pubmed: 13678871.

4. Ponikowski P, Voors A, Anker S, et al. ESC Guidelines for the diagnosis and treatment of acute and chronic heart failure: The Task Force for thediagnosis and treatment of acute and chronic heart failure of the European Society of Cardiology (ESC). Developed with the special contribution of the Heart Failure Association (HFA) of the ESC. Eur Heart J. 2016; 37(27): 2129-2200, doi: 10.1093/eurheartj/ehw128.

5. Gómez OI, Ferrero-Gregori A, Varela RA, et al. Mid-range ejection fraction does not permit risk stratification among patients hospitalized for heart failure. Rev Esp Card., doi: 10.1016/j. recesp.2016.08.019.

6. Varela Romén A, Grigorian Shamagian L, Bandin Diéguez MA, et al. Influence of sex on mortality in hospitalized patients with congestive heart failure and preserved or depressed systolic function. Revista Española de Cardiología (English Edition). 2005; 58(10): 1171-1180, doi: 10.1016/s1885-5857(06)60396-4.

7. Basuray A, French B, Ky B, et al. Heart failure with recovered ejection fraction: clinical description, biomarkers, and outcomes. Circulation. 2014; 129(23): 2380-2387, doi: 10.1161/CIRCULATIONAHA.113.006855, indexed in Pubmed: 24799515.

8. Stevenson LW. Heart failure with better ejection fraction: a modern diagnosis. Circulation. 2014; 129(23): 2364-2367, doi: 10.1161/CIRCULATIONAHA.114.010194, indexed in Pubmed: 24799514 .

9. Mendez GF, Cowie MR. The epidemiological features of heart failure in developing countries: a review of the literature. Int J Cardiol. 2001; 80(2-3): 213-219, indexed in Pubmed: 11578717.

10. MacIntyre K, Capewell S, Stewart S, et al. Evidence of improving prognosis in heart failure: trends in case fatality in 66547 patients hospitalized between 1986 and 1995. Circulation. 2000; 102(10): 1126-1131, indexed in Pubmed: 10973841.

11. Kalogeropoulos AP, Fonarow GC, Georgiopoulou V, et al. Characteristics and Outcomes of Adult Outpatients With Heart Failure and Improved or Recovered Ejection Fraction. JAMA Cardiol. 2016; 1(5): 510-518, doi: 10.1001/jamacardio.2016.1325, indexed in Pubmed: 27434402.

12. Florea VG, Rector TS, Anand IS, et al. Heart failure with improved ejection fraction: clinical characteristics, correlates of recovery, and survival: results from the valsartan heart failure trial. Circ Heart Fail. 2016; 9(7), doi: 10.1161/CIRCHEARTFAILURE.116.003123, indexed in Pubmed: 27413037.

13. Lupón J, Díez-López C, de Antonio M, et al. Recovered heart failure with reduced ejection fraction and outcomes: a prospective study. Eur J Heart Fail. 2017 [Epub ahead of print], doi: 10.1002/ ejhf.824, indexed in Pubmed: 28387002.

14. Nadruz W, West E, Santos M, et al. Heart Failure and Midrange Ejection Fraction: Implications of Recovered Ejection Fraction for Exercise Tolerance and Outcomes. Circ Heart Fail. 2016; 9(4): e002826, doi: 10.1161/CIRCHEARTFAILURE.115.002826, indexed in Pubmed: 27009553.

15. Lang RM, Badano LP, Mor-Avi V, et al. Recommendations for cardiac chamber quantification by echocardiography in adults: an update from the American Society of Echocardiography and the European Association of Cardiovascular Imaging. J Am Soc Echocardiogr. 2015; 28(1): 1-39.e14, doi: 10.1016/j.echo.2014.10.003, indexed in Pubmed: 25559473.

16. Punnoose LR, Givertz MM, Lewis EF, et al. Heart failure with recovered ejection fraction: a distinct clinical entity. J Card Fail. 2011; 17(7): 527-532, doi: 10.1016/j.cardfail.2011.03.005, indexed in Pubmed: 21703523.

17. Moon J, Ko YG, Chung N, et al. Recovery and recurrence of left ventricular systolic dysfunction in patients with idiopathic dilated cardiomyopathy. Can J Cardiol. 2009; 25(5): e147-e150, indexed in Pubmed: 19417864.

18. Waagstein F, Caidahl K, Wallentin I, et al. Long-term beta-blockade in dilated cardiomyopathy. Effects of short- and long-term metoprolol treatment followed by withdrawal and readministration of metoprolol. Circulation. 1989; 80(3): 551-563, indexed in Pubmed: 2548768.

19. Kotecha D, Manzano L, Krum H, et al. Beta-Blockers in Heart Failure Collaborative Group, Meta-Analysis Global Group in Chronic Heart Failure (MAGGIC), Meta-Analysis Global Group in Chronic Heart Failure MAGGIC, Beta-Blockers in Heart Failure Collaborative Group, Meta-analysis Global Group in Chronic Heart Failure (MAGGIC), Meta-Analysis Global Group in Chronic 
Heart Failure, Meta-Analysis Global Group in Chronic Heart Failure, MAGGIC Investigators, Meta-analysis Global Group in Chronic Heart Failure (MAGGIC) Investigators, Meta-Analysis Global Group In Chronic Heart Failure (MAGGIC), Meta-analysis Global Group in Chronic Heart Failure (MAGGIC). The survival of patients with heart failure with preserved or reduced left ventricular ejection fraction: an individual patient data metaanalysis. Eur Heart J. 2012; 33(14): 1750-1757, doi: 10.1093/ eurhearti/ehr254, indexed in Pubmed: 21821849.

20. Wilcox JE, Fonarow GC, Yancy CW, et al. Factors associated with improvement in ejection fraction in clinical practice among patients with heart failure: findings from IMPROVE HF. Am Heart J. 2012; 163(1): 49-56.e2, doi: 10.1016/j.ahj.2011.10.001, indexed in Pubmed: 22172436.

21. Tian Y, Zhang P, Li X, et al. True complete left bundle branch block morphology strongly predicts good response to cardiac resynchronization therapy. Europace. 2013; 15(10): 1499-1506, doi: 10.1093/europace/eut049, indexed in Pubmed: 23468351.

22. Hsu JC, Solomon SD, Bourgoun M, et al. MADIT-CRT Executive Committee. Predictors of super-response to cardiac resynchronization therapy and associated improvement in clinical outcome: the MADIT-CRT (multicenter automatic defibrillator implantation trial with cardiac resynchronization therapy) study. J Am Coll Cardiol. 2012; 59(25): 2366-2373, doi: 10.1016/j. jacc.2012.01.065, indexed in Pubmed: 22698490.

23. Gradman A, Deedwania P, Cody R, et al. Predictors of total mortality and sudden death in mild to moderate heart failure. J Am Coll Cardiol. 1989; 14(3): 564-570, doi: 10.1016/0735-1097(89)90093-4.

24. Brophy JM, Dagenais GR, McSherry F, et al. A multivariate model for predicting mortality in patients with heart failure and systolic dysfunction. Am J Med. 2004; 116(5): 300-304, doi: 10.1016/j. amjmed.2003.09.035, indexed in Pubmed: 14984814.

25. Eichhor EJ. Prognosis determination in heart failure. Am J Med. 2001; 110(Suppl 7A): 14S-36S.
26. Yancy CW, Jessup M, Bozkurt B, et al. 2013 ACCF/AHA guideline for the management of heart failure: a report of the American College of Cardiology Foundation/ American Heart Association Task Force on Practice Guidelines. J Am Coll Cardiol. 2013; 62: $147-239$.

27. Amorim S, Campelo M, Martins E, et al. Prevalence, predictors and prognosis of ventricular reverse remodeling in idiopathic dilated cardiomyopathy. Rev Port Cardiol. 2016; 35(5): 253-260, doi: 10.1016/j.repc.2015.11.014, indexed in Pubmed: 27118094.

28. Pfeffer MA, Swedberg K, Granger CB, et al. CHARM Investigators and Committees. Effects of candesartan on mortality and morbidity in patients with chronic heart failure: the CHARM-Overall programme. Lancet. 2003; 362(9386): 759-766, indexed in Pubmed: 13678868.

29. Svanström H, Pasternak B, Melbye M, et al. Use of different types of angiotensin converting enzyme inhibitors and mortality in systolic heart failure. Int J Cardiol. 2015; 182: 90-96, doi: 10.1016/j.ijcard.2014.12.092, indexed in Pubmed: 25576729.

30. Kramer DG, Trikalinos TA, Kent DM, et al. Quantitative evaluation of drug or device effects on ventricular remodeling as predictors of therapeutic effects on mortality in patients with heart failure and reduced ejection fraction: a meta-analytic approach. J Am Coll Cardiol. 2010; 56(5): 392-406, doi: 10.1016/j. jacc.2010.05.011, indexed in Pubmed: 20650361.

31. Bonow RO, Maurer G, Lee KL, et al. STICH Trial Investigators. Myocardial viability and survival in ischemic left ventricular dysfunction. N Engl J Med. 2011; 364(17): 1617-1625, doi: 10.1056/ NEJMoa1100358, indexed in Pubmed: 21463153.

32. Moss AJ, Hall WJ, Cannom DS, et al. Improved survival with an implanted defibrillator in patients with coronary disease at high risk for ventricular arrhythmia. Multicenter Automatic Defibrillator Implantation Trial Investigators. N Engl J Med. 1996; 335(26): 1933-1940, doi: 10.1056/NEJM199612263352601, indexed in Pubmed: 8960472. 